\title{
Asymptotic Stabilization of a Five-link, Four-Actuator, Planar Bipedal Runner
}

\author{
C. Chevallereau \\ IRCCyN, Ecole Centrale de Nantes \\ UMR CNRS 6597, BP 92101 \\ 1 rue de la Noë, 44321 Nantes \\ cedex 03, France \\ E-mail: Christine.Chevallereau@irccyn.ec-nantes.fr
}

\author{
E.R. Westervelt \\ Department of Mechanical Engineering \\ The Ohio State University \\ Columbus, Ohio 43210, USA \\ E-mail: westervelt.4@osu.edu
}

\author{
J.W. Grizzle \\ Control Systems Laboratory \\ EECS Department \\ University of Michigan \\ Ann Arbor, Michigan 48109-2122, USA \\ E-mail: grizzle@umich.edu
}

\begin{abstract}
Provably asymptotically-stable running-gaits are developed for the five-link, four-actuator bipedal robot, RABBIT. A controller is designed so that the Poincaré return map associated with the running gait can be computed on the basis of a model with impulse-effects that, perviously, had been used only for the design of walking gaits. This feedback design leads to the notion of a hybrid zero dynamics (HZD) for running and to the closed-form computation of the Poincaré return map on the zero dynamics. The main theorem is illustrated via simulation. Animations of the resulting running motion are available on the web.
\end{abstract}

\section{INTRODUCTION}

This paper addresses the design and analysis of asymptotically stable running gaits for RABBIT, a five-link, fouractuator, planar, revolute-jointed, bipedal robot [1]. In a series of papers, the authors and colleagues have developed new feedback control strategies [2]-[7] that achieve provably asymptotically-stable walking gaits in underactuated bipeds, such as RABBIT, and demonstrated many of them experimentally [8], [9]. In regards to running, open-loop trajectories have been studied in [10], [11]. An objective of this paper is to develop a time-invariant feedback controller that realizes these open-loop running trajectories as provably asymptoticallystable orbits. A key step is to design the feedback controller in such a way that the Poincaré return map associated with a running gait can be computed on the basis of the models with impulse effects studied in [3] for the design of walking gaits. This leads to the notion of a hybrid zero dynamics (HZD) for running, and to the closed-form computation of a restricted Poincaré map. The main results are illustrated via simulation. The reader may wish to view animations and other supporting material that is available on the web [12].

\section{RELATED WORK}

In the early 1980's, Raibert proposed an elegant conceptualization of running in terms of a one-legged, prismatic-kneed hopper [13], [14]. He decomposed his control actions into three parts-hopping height, foot touchdown angle, and body posture - and emphasized the role of symmetry in designing stable running motions. The remarkable success of Raibert's control law motivated others to analytically characterize its stability [15]-[17], and to further investigate the role of passive elements in achieving efficient running with a hopper [18]. Raibert's control scheme has been augmented with leg-coordination logic to achieve running in prismatic-kneed bipeds and quadrupeds [13], [19].

In 2003, both Iguana Robotics and Sony announced (separate) experimental demonstrations of running for bipedal robots with revolute knees. Apparently, the controller of the Sony robot is based on a common heuristic called the zero moment point (ZMP) criterion, and that of Iguana Robotics is based on central pattern generators (CPGs). To our best knowledge, only two other bipeds with revolute knees have been designed to perform running-Jogging Johnnie in Munich [20], [21] and RABBIT in Grenoble [1], [22] - and running has not yet been attempted on either one.

\section{Mechanical Model of A Biped Runner}

\section{A. The biped}

The studied bipedal robot evolves in the sagittal plane with respect to a flat surface; see Fig. 1. The flat surface will be referred to as the ground. The robot is composed of five rigid links with mass, connected through ideal (i.e., rigid and frictionless) revolute joints to form a torso and two identical legs, with each leg articulated by a knee. Each leg end is terminated in a point so that, in particular, the robot does not have feet.

The robot is said to be in flight phase when there is no contact with the ground, and in stance phase when one leg end is in stationary contact with the ground (that is, the leg end is acting as an ideal pivot) and the other leg is free. For the stance phase, the leg in contact with the ground is called the stance leg and the other leg is the swing leg.

\section{B. Lagrangian model for flight}

A convenient choice of configuration variables is depicted in Fig. 1. The vector of body coordinates $q_{\mathrm{b}}$ consisting of the relative angles $\left(q_{1}, q_{2}, q_{3}, q_{4}\right)^{\prime}$ describes the shape of the biped. The biped's absolute orientation with respect to the world frame is given by $q_{5}$. The biped's absolute position is specified by the Cartesian coordinates of the center of mass, 


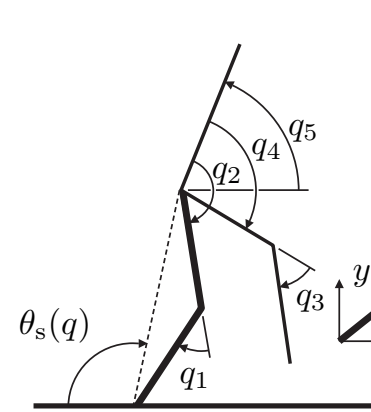

(a)

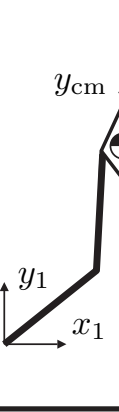

(b)

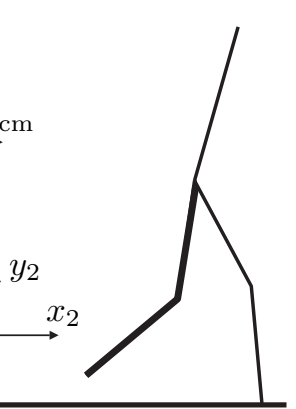

(c)
Fig. 1. Different phases of running with coordinate conventions labeled. The robot is shown (a) at the end of the stance phase; (b) during flight; and (c) at the beginning of the stance phase just before impact. To avoid clutter, the coordinate conventions have been spread out over the single support and flight phases even though they apply to all three phases. leg- 1 is presented in bold. Angles are positive in the clockwise direction.

$\left(x_{\mathrm{cm}}, y_{\mathrm{cm}}\right)$. The vector of generalized coordinates is denoted as $q_{\mathrm{f}}:=\left(q_{\mathrm{b}}^{\prime}, q_{5}, x_{\mathrm{cm}}, y_{\mathrm{cm}}\right)^{\prime}$.

A dynamic model,

$$
D_{\mathrm{f}}\left(q_{\mathrm{b}}\right) \ddot{q}_{\mathrm{f}}+C_{\mathrm{f}}\left(q_{\mathrm{b}}, \dot{q}_{\mathrm{f}}\right) \dot{q}_{\mathrm{f}}+G_{\mathrm{f}}\left(q_{\mathrm{f}}\right)=B_{\mathrm{f}} u
$$

is easily obtained with the method of Lagrange where $D_{\mathrm{f}}$ is the inertia matrix, the matrix $C_{\mathrm{f}}$ contains Coriolis and centrifugal terms, and $G_{\mathrm{f}}$ is the gravity vector. In these coordinates, the inertia matrix has the special form

$$
D_{\mathrm{f}}=\left[\begin{array}{cc}
A\left(q_{\mathrm{b}}\right) & 0_{5 \times 2} \\
0_{2 \times 5} & m I_{2 \times 2}
\end{array}\right],
$$

where $m$ is the total mass of the robot and $A$ depends only on $q_{\mathrm{b}}$, because the total kinetic energy is invariant under rotations and translations of the body. The principle of virtual work yields that the external torques are

$$
B_{\mathrm{f}} u=\left[\begin{array}{c}
I_{4 \times 4} \\
0_{3 \times 4}
\end{array}\right] u,
$$

where $u$ is the vector of actuator torques applied at the four joints of the robot.

Introducing the state vector $x_{\mathrm{f}}:=\left(q_{\mathrm{f}}^{\prime}, \dot{q}_{\mathrm{f}}^{\prime}\right)$, the Lagrangian model (1) is easily expressed as

$$
\dot{x}_{\mathrm{f}}=f_{\mathrm{f}}\left(x_{\mathrm{f}}\right)+g_{\mathrm{f}}\left(x_{\mathrm{f}}\right) u \text {. }
$$

The state space is taken as $T \mathcal{Q}_{\mathrm{f}}:=\left\{x_{\mathrm{f}}:=\left(q_{\mathrm{f}}^{\prime}, \dot{q}_{\mathrm{f}}^{\prime}\right)^{\prime} \mid q_{\mathrm{f}} \in\right.$ $\left.\mathcal{Q}_{\mathrm{f}}, \dot{q}_{\mathrm{f}} \in \mathbb{R}^{7}\right\}$, where the configuration space $\mathcal{Q}_{\mathrm{f}}$ is a simplyconnected, open subset of $(-\pi, \pi)^{5} \times \mathbb{R}^{2}$ corresponding to physically reasonable configurations of the robot.

\section{Lagrangian model for stance}

For the stance phase, the generalized coordinates can be taken as $q:=\left(q_{\mathrm{b}}^{\prime}, q_{5}\right)^{\prime}=\left(q_{1}, \cdots, q_{5}\right)^{\prime}$. Since the robot's legs are identical, in the stance phase, it will be assumed without loss of generality that leg- 1 is in contact with the ground. Moreover, the Cartesian position of the stance leg end will be identified with the origin of the $(x-y)$-axes of a world frame.
The position of the center of mass can be expressed in terms of $q$ per

$$
\left[\begin{array}{l}
x_{\mathrm{cm}}(q) \\
y_{\mathrm{cm}}(q)
\end{array}\right]=f_{1}(q),
$$

where $f_{1}$ is determined from the robot's geometric parameters (link lengths, masses, positions of the centers of mass). Hence

$$
\dot{q}_{\mathrm{f}}=\left[\begin{array}{c}
I_{5 \times 5} \\
\frac{\partial f_{1}}{\partial q}
\end{array}\right] \dot{q} .
$$

The method of Lagrange results in

$$
D_{\mathrm{s}}\left(q_{\mathrm{b}}\right) \ddot{q}+C_{\mathrm{s}}\left(q_{\mathrm{b}}, \dot{q}\right) \dot{q}+G_{\mathrm{s}}(q)=B_{\mathrm{s}} u,
$$

where,

$$
D_{\mathrm{s}}\left(q_{\mathrm{b}}\right)=A\left(q_{\mathrm{b}}\right)+m \frac{\partial f_{1}(q)^{\prime}}{\partial q} \frac{\partial f_{1}(q)}{\partial q}
$$

and

$$
B_{\mathrm{s}} u=\left[\begin{array}{c}
I_{4 \times 4} \\
0
\end{array}\right] u \text {. }
$$

Because the kinetic energy is invariant under rotations of the body, $D_{\mathrm{s}}$ depends only on $q_{\mathrm{b}}$.

Introducing the state vector $x_{\mathrm{s}}:=\left(q^{\prime}, \dot{q}^{\prime}\right)$, the Lagrangian model (7) is easily expressed as

$$
\dot{x}_{\mathrm{s}}=f_{\mathrm{s}}\left(x_{\mathrm{s}}\right)+g_{\mathrm{s}}\left(x_{\mathrm{s}}\right) u \text {. }
$$

The state space is taken as $T \mathcal{Q}_{\mathrm{s}}:=\left\{x_{\mathrm{s}}:=\left(q^{\prime}, \dot{q}^{\prime}\right)^{\prime} \mid q \in\right.$ $\left.\mathcal{Q}_{\mathrm{s}}, \quad \dot{q} \in \mathbb{R}^{5}\right\}$, where the configuration space $\mathcal{Q}_{\mathrm{s}}$ is a simply-connected, open subset of $(-\pi, \pi)^{5}$ corresponding to physically reasonable configurations.

\section{The impact model}

The Cartesian position of the end of leg- 2 can be expressed in terms of the Cartesian position of the center of mass and the robot's angular coordinates as

$$
\left[\begin{array}{l}
x_{2} \\
y_{2}
\end{array}\right]=\left[\begin{array}{l}
x_{\mathrm{cm}} \\
y_{\mathrm{cm}}
\end{array}\right]-f_{2}(q),
$$

where $f_{2}$ is determined from the robot's parameters (links lengths, masses, positions of the centers of mass); see (5). When leg-2 touches the ground at the end of a flight phase, an impact takes place. The impact model of [23] is used, which represents the ground reaction forces at impact as impulses. The impact is assumed inelastic, with the velocity of the contact leg end becoming zero instantaneously; furthermore, after impact, the contact leg end is assumed to act as an ideal pivot. This model yields that the robot's configuration $q_{\mathrm{f}}$ is unchanged during impact, and there are instantaneous changes in the velocities. The velocity vectors just before and just after impact, are denoted $\dot{q}_{\mathrm{f}}^{-}, \dot{q}^{-}$and $\dot{q}_{\mathrm{f}}^{+}, \dot{q}^{+}$respectively.

The robot's angular velocity vector after impact is given by

$$
\dot{q}^{+}=\left[A+m \frac{\partial f_{2}{ }^{\prime}}{\partial q} \frac{\partial f_{2}}{\partial q}\right]^{-1}\left(A \dot{q}^{-}+m \frac{\partial f_{2}^{\prime}}{\partial q}\left[\begin{array}{c}
\dot{x}_{\mathrm{cm}}^{-} \\
\dot{y}_{\mathrm{cm}}^{-}
\end{array}\right]\right)
$$

which, for later use, is written as

$$
\dot{q}^{+}=\tilde{\Delta}\left(q_{\mathrm{f}}^{-}, \dot{q}_{\mathrm{f}}^{-}\right) .
$$




\section{E. Some linear and angular momentum relationships}

A few linear and angular momentum properties of the mechanical models are noted. Let $\sigma_{\mathrm{cm}}$ denote the angular momentum of the biped about its center of mass. In the flight phase, $\sigma_{\mathrm{cm}}$ can be computed by $\sigma_{\mathrm{cm}}=\frac{\partial K_{\mathrm{f}}}{\partial \dot{q}_{5}}=A_{5} \dot{q}$, where $A_{5}$ is the fifth row of $A$. The model (1) yields conservation of $\sigma_{\mathrm{cm}}$

$$
\dot{\sigma}_{\mathrm{cm}}=0
$$

in addition,

$$
\ddot{x}_{\mathrm{cm}}=0 \quad \text { and } \quad \ddot{y}_{\mathrm{cm}}=-g \text {, }
$$

which correspond to linear momentum conservation.

Let $\sigma_{i}$ denote the angular momentum of the biped about the end of leg-i, for $i=1,2$. The three angular momenta are related by

$$
\sigma_{i}=\sigma_{\mathrm{cm}}+m\left(\left(y_{\mathrm{cm}}-y_{i}\right) \dot{x}_{\mathrm{cm}}-\left(x_{\mathrm{cm}}-x_{i}\right) \dot{y}_{\mathrm{cm}}\right) .
$$

For the stance phase, $\sigma_{1}$ can de determined by $\sigma_{1}=\frac{\partial K_{\mathrm{s}}}{\partial \dot{q}_{5}}=$ $D_{\mathrm{s}, 5} \dot{q}$, where $D_{\mathrm{s}, 5}$ is the fifth row of $D_{\mathrm{s}}$.

The impact model of [23] yields conservation of angular momentum about the impact point. Since the impact occurs in the flight phase on leg-2 and the stance model assumes the stance leg is leg-1 the conservation of momentum relation is expressed as

$$
\sigma_{1}^{+}=\sigma_{2}^{-} .
$$

\section{Hybrid Model of RUNNING}

The overall biped robot model can be expressed as a nonlinear hybrid system containing two state manifolds (called "charts" in [24]):

$$
\begin{aligned}
& \Sigma_{\mathrm{f}}:\left\{\begin{aligned}
\mathcal{X}_{\mathrm{f}} & =T \mathcal{Q}_{\mathrm{f}} \\
\mathcal{F}_{\mathrm{f}}:\left(\dot{x}_{\mathrm{f}}\right) & =f_{\mathrm{f}}\left(x_{\mathrm{f}}\right)+g_{\mathrm{f}}\left(x_{\mathrm{f}}\right) u \\
S_{\mathrm{f}}^{\mathrm{s}} & =\left\{x_{\mathrm{f}} \in T \mathcal{Q}_{\mathrm{f}} \mid H_{\mathrm{f}}^{\mathrm{s}}\left(x_{\mathrm{f}}\right)=0\right\} \\
\mathcal{T}_{\mathrm{f}}^{\mathrm{s}}: x_{\mathrm{s}}^{+} & =\Delta_{\mathrm{f}}^{\mathrm{s}}\left(x_{\mathrm{f}}^{-}\right)
\end{aligned}\right. \\
& \Sigma_{\mathrm{s}}:\left\{\begin{aligned}
\mathcal{X}_{\mathrm{s}} & =T \mathcal{Q}_{\mathrm{s}} \\
\mathcal{F}_{\mathrm{s}}:\left(\dot{x}_{\mathrm{s}}\right) & =f_{\mathrm{s}}\left(x_{\mathrm{s}}\right)+g_{\mathrm{s}}\left(x_{\mathrm{s}}\right) u \\
S_{\mathrm{s}}^{\mathrm{f}} & =\left\{x_{\mathrm{s}} \in T \mathcal{Q}_{\mathrm{s}} \mid H_{\mathrm{s}}^{\mathrm{f}}\left(x_{\mathrm{s}}\right)=0\right\} \\
\mathcal{T}_{\mathrm{s}}^{\mathrm{f}}: x_{\mathrm{f}}^{+} & =\Delta_{\mathrm{s}}^{\mathrm{f}}\left(x_{\mathrm{s}}^{-}\right)
\end{aligned}\right.
\end{aligned}
$$

where, for example, $\mathcal{F}_{\mathrm{f}}$ is the flow on state manifold $\mathcal{X}_{\mathrm{f}}, S_{\mathrm{f}}^{\mathrm{s}}$ is the switching hyper-surface for transitions between $\mathcal{X}_{\mathrm{f}}$ and $\mathcal{X}_{\mathrm{s}}, \mathcal{T}_{\mathrm{f}}^{\mathrm{s}}: S_{\mathrm{f}}^{\mathrm{s}} \rightarrow \mathcal{X}_{\mathrm{s}}$ is the transition function applied when $x_{\mathrm{f}} \in S_{\mathrm{f}}^{\mathrm{s}}$.

The transition from flight phase to stance phase occurs when leg-2 impacts the ground. Hence, $H_{\mathrm{f}}^{\mathrm{s}}\left(x_{\mathrm{f}}\right)=y_{2}$; recall (11). The ensuing initial value of the stance phase, $x_{\mathrm{s}}^{+}$, is determined from the impact model of Section III-D. A relabeling matrix $R$ is applied to the angular coordinates to account for the impact occurring on leg- 2 while the stance model assumes leg- 1 is in contact with the ground:

$$
\Delta_{\mathrm{f}}^{\mathrm{s}}\left(x_{\mathrm{f}}^{-}\right)=\left[\begin{array}{l}
R q^{-} \\
R \tilde{\Delta}\left(x_{\mathrm{f}}^{-}\right)
\end{array}\right],
$$

where (13) has been used.

The transition from stance phase to flight phase can be initiated by causing the acceleration of the stance leg end to become positive. If torque discontinuities ${ }^{1}$ are allowedas they are assumed to be in this paper-when to transition into the flight phase becomes a control decision. Here, in view of simplifying the analysis of periodic orbits in Section VI, the transition is assumed to occur at a fixed point in the stance phase. Hence, $H_{\mathrm{s}}^{\mathrm{f}}=\theta_{\mathrm{s}}(q)-\theta_{\mathrm{s}}^{-}$, where $\theta_{\mathrm{s}}(q):=\frac{q_{1}}{2}+q_{2}+q_{5}$ is the angle of the hips with respect to end of the stance leg (see Fig. 1) and $\theta_{\mathrm{s}}^{-}$is a constant to be determined. The ensuing initial value of the flight phase, $x_{\mathrm{f}}^{+}$, is defined so as to achieve continuity in the position and velocity variables, using (5) and (6):

$$
\Delta_{\mathrm{s}}^{\mathrm{f}}\left(x_{\mathrm{s}}^{-}\right)=\left[\begin{array}{l}
\left\{\begin{array}{l}
q^{-} \\
f_{1}\left(q^{-}\right)
\end{array}\right. \\
\left\{\begin{array}{l}
\dot{q}^{-} \\
\frac{\partial f_{1}\left(q^{-}\right)}{\partial q} \dot{q}^{-}
\end{array}\right] .
\end{array}\right.
$$

Continuity of the torques is not imposed, and hence neither is continuity of the accelerations. It is assumed that the control law in the flight phase will be designed to achieve $\ddot{y}_{2}^{+}>0$; see [11].

\section{Control Law Development}

\section{A. Stance phase control}

As in $[3$, Sec. V], define the output

$$
y_{\mathrm{s}}=h_{\mathrm{s}}(q):=q_{\mathrm{b}}-h_{d, \mathrm{~s}} \circ \theta_{\mathrm{s}}(q)
$$

on (7), where the twice continuously differentiable function $h_{d, \mathrm{~s}}: \mathbb{R} \rightarrow \mathbb{R}^{4}$ encodes the stance-phase gait. It is assumed that the associated decoupling matrix is invertible, $\Phi_{\mathrm{s}}(q):=$ $\left[h_{\mathrm{s}}^{\prime}, \theta_{\mathrm{s}}\right]^{\prime}$ is a diffeomorphism,

$$
Z_{\mathrm{s}}:=\left\{x_{\mathrm{s}} \in T \mathcal{Q}_{\mathrm{s}} \mid h_{\mathrm{s}}\left(x_{\mathrm{s}}\right)=0, L_{f_{\mathrm{s}}} h_{\mathrm{s}}\left(x_{\mathrm{s}}\right)=0\right\}
$$

is an embedded two-dimensional submanifold of $T \mathcal{Q}_{\mathrm{s}}$, and, $S_{\mathrm{s}}^{\mathrm{f}} \cap Z_{\mathrm{s}}$ is an embedded one-dimensional submanifold of $T \mathcal{Q}_{\mathrm{s}}$.

The feedback control is chosen to be continuous and to render $Z_{\mathrm{s}}$ invariant under the closed-loop dynamics as well as attractive in finite-time (the exact hypotheses are $\mathrm{CH} 2-\mathrm{CH} 5$ in [3, IV.C]):

$$
\begin{aligned}
u_{\mathrm{s}}\left(x_{\mathrm{s}}\right)=\left(L_{g_{\mathrm{s}}} L_{f_{\mathrm{s}}} h_{\mathrm{s}}\left(x_{\mathrm{s}}\right)\right)^{-1}\left(v \left(h_{\mathrm{s}}\left(x_{\mathrm{s}}\right)\right.\right. & \left., L_{f_{\mathrm{s}}} h_{\mathrm{s}}\left(x_{\mathrm{s}}\right)\right) \\
& \left.-L_{f_{\mathrm{s}}}^{2} h_{\mathrm{s}}\left(x_{\mathrm{s}}\right)\right),
\end{aligned}
$$

where $v$ renders the origin of $\ddot{y}_{\mathrm{s}}=v$ globally asymptotically stable with finite-convergence time. The closed-loop system is denoted

$$
f_{\mathrm{cl}, \mathrm{s}}\left(x_{\mathrm{s}}\right):=f_{\mathrm{s}}\left(x_{\mathrm{s}}\right)+g_{\mathrm{s}}\left(x_{\mathrm{s}}\right) u_{\mathrm{s}}\left(x_{\mathrm{s}}\right) .
$$

The feedback control

$$
u_{\mathrm{s}}^{*}\left(x_{\mathrm{s}}\right)=-\left(L_{g_{\mathrm{s}}} L_{f_{\mathrm{s}}} h_{\mathrm{s}}\left(x_{\mathrm{s}}\right)\right)^{-1} L_{f_{\mathrm{s}}}^{2} h_{\mathrm{s}}\left(x_{\mathrm{s}}\right)
$$

\footnotetext{
${ }^{1}$ This is a modeling decision. In practice, the torque is continuous due to actuator dynamics. It is assumed here that the actuator time constant is small enough that it need not be modeled.
} 
renders $Z_{\mathrm{s}}$ invariant under the stance-phase dynamics; that is, for every $z \in Z_{\mathrm{s}}$,

$$
f_{\text {zero }}(z):=f_{\mathrm{s}}(z)+g_{\mathrm{s}}(z) u_{\mathrm{s}}^{*}(z) \in T_{z} Z_{\mathrm{s}} .
$$

$Z_{\mathrm{s}}$ is called the stance-phase zero dynamics manifold and $\dot{z}=$ $f_{\text {zero }}(z)$ is called the stance-phase zero dynamics. Following the development in [1], [3], $\left(\theta_{\mathrm{s}}, \sigma_{1}\right)$ is a valid set of local coordinates for $Z_{\mathrm{s}}$ and in these coordinates the zero dynamics has the form

$$
\begin{aligned}
& \dot{\theta}_{\mathrm{s}}=\frac{1}{I\left(\theta_{\mathrm{s}}\right)} \sigma_{1}, \\
& \dot{\sigma}_{1}=m g x_{\mathrm{cm}}\left(\theta_{\mathrm{s}}\right),
\end{aligned}
$$

where $I\left(\theta_{\mathrm{S}}\right)$ plays the role of an inertia. Moreover, in these coordinates, $S_{\mathrm{s}}^{\mathrm{f}} \cap Z_{\mathrm{s}}$ is given by

$$
\left\{\left(q_{0}^{-}, \dot{q}^{-}\right) \mid q_{0}^{-}=\Phi_{\mathrm{s}}^{-1}\left(0, \theta_{\mathrm{s}}^{-}\right), \quad \dot{q}^{-}=\dot{q}_{0}^{-} \sigma_{1}^{-}, \quad \sigma_{1}^{-} \in \mathbb{R}\right\},
$$

where

$$
\dot{q}_{0}^{-}=\left.\left[\begin{array}{c}
\frac{\partial h_{\mathrm{s}}}{\partial q} \\
A_{5}
\end{array}\right]^{-1}\right|_{q=q_{0}^{-}}\left[\begin{array}{l}
0 \\
1
\end{array}\right] .
$$

For later use in computing a Poincaré return map on the zero dynamics, it is noted that (27) has Lagrangian [3, Eq. (59)] $\mathcal{L}_{\text {zero }}:=K_{\text {zero }}-V_{\text {zero }}$, where

$$
\begin{aligned}
K_{\text {zero }} & :=\frac{1}{2}\left(\sigma_{1}\right)^{2} \\
V_{\text {zero }}\left(\theta_{\mathrm{s}}\right) & :=-\int_{\theta_{\mathrm{s}}^{+}}^{\theta_{\mathrm{s}}} I(\xi) m g x_{\mathrm{cm}}(\xi) d \xi ;
\end{aligned}
$$

the choice of the lower limit $\theta_{\mathrm{s}}^{+}$is arbitrary and will be selected later. Also for later use, define

$$
\left[\begin{array}{c}
\lambda_{x}\left(q_{0}^{-}\right) \\
\lambda_{y}\left(q_{0}^{-}\right)
\end{array}\right]:=\frac{\partial f_{1}\left(q_{0}^{-}\right)}{\partial q} \dot{q}_{0}^{-},
$$

so that

$$
\left.\left[\begin{array}{c}
\dot{x}_{\mathrm{cm}}^{-} \\
\dot{y}_{\mathrm{cm}}^{-}
\end{array}\right]\right|_{S_{\mathrm{s}}^{\mathrm{f} \cap Z_{\mathrm{s}}}}=\left[\begin{array}{c}
\lambda_{x}\left(q_{0}^{-}\right) \\
\lambda_{y}\left(q_{0}^{-}\right)
\end{array}\right] \sigma_{1}^{-} .
$$

\section{B. Flight phase control}

The overall goal of the flight-phase controller is to land the robot in a favorable manner for continuing with the stance phase. It will turn out that a particularly interesting objective is the following: if the robot enters the flight phase from the stance-phase zero dynamics manifold, $Z_{\mathrm{s}}$, control the robot so that it lands on $Z_{\mathrm{s}}$ in a fixed configuration. The analytical motivation for this objective will be made clear in Section VI. The feasibility of landing in a fixed configuration will be illustrated in Section VII with a feedback controller that depends on $x_{\mathrm{f}}$ and the final value of the state of the preceding stance phase. To realize such a controller as a state-variable feedback, the flight-state vector is augmented with dummy variables, $\dot{a}_{\mathrm{f}}=0$, whose value can be set at the transition from stance to flight. Let $a_{\mathrm{f}} \in \mathcal{A}:=\mathbb{R}^{\operatorname{dim}\left(a_{\mathrm{f}}\right)}$.

In other regards, paralleling the development of the stance phase controller, define the output

$$
y_{\mathrm{f}}=h_{\mathrm{f}}\left(q_{\mathrm{f}}, a_{\mathrm{f}}\right):=q_{\mathrm{b}}-h_{d, \mathrm{f}}\left(x_{\mathrm{cm}}, a_{\mathrm{f}}\right),
$$

where $h_{d, \mathrm{f}}$ is at least twice differentiable. Then the following can be easily shown: for any value of $a_{\mathrm{f}}$,

1) the decoupling matrix, $L_{g_{\mathrm{f}}} L_{f_{\mathrm{f}}} h_{\mathrm{f}}$, is everywhere invertible;

2) $\Phi_{\mathrm{f}}:=\left[h_{\mathrm{f}}^{\prime}, q_{5}, x_{\mathrm{cm}}, y_{\mathrm{cm}}\right]^{\prime}$ is a global diffeomorphism on $\mathcal{Q}_{\mathrm{f}}$

3) the flight-phase zero-dynamics manifold,

$$
Z_{\mathrm{f}}:=\left\{x_{\mathrm{f}} \in T \mathcal{Q}_{\mathrm{f}} \mid h_{\mathrm{f}}\left(x_{\mathrm{f}}, a_{\mathrm{f}}\right)=0, L_{f_{\mathrm{f}}} h_{\mathrm{f}}\left(x_{\mathrm{f}}, a_{\mathrm{f}}\right)=0\right\},
$$

is a six-dimensional embedded submanifold of $T \mathcal{Q}_{\mathrm{f}}$;

4) $S_{\mathrm{f}}^{\mathrm{s}} \cap Z_{\mathrm{f}}$ is a five-dimensional embedded submanifold of $T \mathcal{Q}_{\mathrm{f}}$;

5) $\left(q_{5}, x_{\mathrm{cm}}, y_{\mathrm{cm}}, \sigma_{\mathrm{cm}}, \dot{x}_{\mathrm{cm}}, \dot{y}_{\mathrm{cm}}\right)$ is a set of global coordinates for $Z_{\mathrm{f}}$; and

6) the flight-phase zero dynamics is given by (14), (15) and

$$
\dot{q}_{5}=\kappa_{1, \mathrm{f}}\left(\sigma_{\mathrm{cm}}, x_{\mathrm{cm}}, \dot{x}_{\mathrm{cm}}, a_{\mathrm{f}}\right)
$$

where (36) arises from evaluating

$$
\dot{q}_{5}=\frac{\sigma_{\mathrm{cm}}}{A_{55}\left(q_{\mathrm{b}}\right)}-\sum_{i=1}^{4} \frac{A_{5 i}\left(q_{\mathrm{b}}\right)}{A_{55}\left(q_{\mathrm{b}}\right)} \dot{q}_{i}
$$

on $Z_{\mathrm{f}}$.

The feedback controller is defined as

$$
\begin{aligned}
u_{\mathrm{f}}\left(x_{\mathrm{f}}, a_{\mathrm{f}}\right):=- & \left(L_{g_{\mathrm{f}}} L_{f_{\mathrm{f}}} h_{\mathrm{f}}\left(x_{\mathrm{f}}, a_{\mathrm{f}}\right)\right)^{-1}\left(K_{p} h_{\mathrm{f}}\left(x_{\mathrm{f}}, a_{\mathrm{f}}\right)\right. \\
& \left.+K_{d} L_{f_{\mathrm{f}}} h_{\mathrm{f}}\left(x_{\mathrm{f}}, a_{\mathrm{f}}\right)+L_{f_{\mathrm{f}}}^{2} h_{\mathrm{f}}\left(x_{\mathrm{f}}, a_{\mathrm{f}}\right)\right),
\end{aligned}
$$

where $\ddot{y}_{\mathrm{f}}+K_{d} \dot{y}_{\mathrm{f}}+K_{p} y_{\mathrm{f}}=0$ is exponentially stable. Let $\bar{x}_{\mathrm{f}}:=\left(x_{\mathrm{f}}^{\prime}, a_{\mathrm{f}}^{\prime}\right)^{\prime}$ and denote the right-hand side of (4) and the dummy variabls $\dot{a}_{\mathrm{f}}=0$ in closed loop with (38) by

$$
f_{\mathrm{cl}, \mathrm{f}}\left(\bar{x}_{\mathrm{f}}\right):=\left[\begin{array}{l}
f_{\mathrm{f}}\left(x_{\mathrm{f}}\right)+g_{\mathrm{f}}\left(x_{\mathrm{f}}\right) u_{\mathrm{f}}\left(\bar{x}_{\mathrm{f}}\right) \\
0
\end{array}\right] .
$$

\section{Closed-loop hybrid model}

The closed-loop hybrid model is

$$
\begin{aligned}
& \Sigma_{\mathrm{f}}:\left\{\begin{aligned}
\overline{\mathcal{X}}_{\mathrm{f}} & =T \mathcal{Q}_{\mathrm{f}} \times \mathcal{A} \\
\overline{\mathcal{F}}_{\mathrm{cl}, \mathrm{f}}:\left(\dot{\bar{x}}_{\mathrm{f}}\right) & =\bar{f}_{\mathrm{cl}, \mathrm{f}}\left(\bar{x}_{\mathrm{f}}\right) \\
\bar{S}_{\mathrm{f}}^{\mathrm{s}} & =\left\{\left(x_{\mathrm{f}}, a_{\mathrm{f}}\right) \in \overline{\mathcal{X}}_{\mathrm{f}} \mid H_{\mathrm{f}}^{\mathrm{s}}\left(x_{\mathrm{f}}\right)=0\right\} \\
\overline{\mathcal{T}}_{\mathrm{f}}^{\mathrm{s}}: x_{\mathrm{s}}^{+} & =\bar{\Delta}_{\mathrm{f}}^{\mathrm{s}}\left(\bar{x}_{\mathrm{f}}^{-}\right):=\Delta_{\mathrm{f}}^{\mathrm{s}}\left(x_{\mathrm{f}}^{-}\right)
\end{aligned}\right. \\
& \Sigma_{\mathrm{s}}:\left\{\begin{aligned}
\mathcal{X}_{\mathrm{s}} & =T \mathcal{Q}_{\mathrm{s}} \\
\mathcal{F}_{\mathrm{cl}, \mathrm{s}}:\left(\dot{x}_{\mathrm{s}}\right) & =f_{\mathrm{cl}, \mathrm{s}}\left(x_{\mathrm{s}}\right) \\
S_{\mathrm{s}}^{\mathrm{f}} & =\left\{x_{\mathrm{s}} \in T \mathcal{Q}_{\mathrm{s}} \mid H_{\mathrm{s}}^{\mathrm{f}}\left(x_{\mathrm{s}}\right)=0\right\} \\
\overline{\mathcal{T}}_{\mathrm{s}}^{\mathrm{f}}: x_{\mathrm{f}}^{+} & =\Delta_{\mathrm{s}}^{\mathrm{f}}\left(x_{\mathrm{s}}^{-}\right), a_{\mathrm{f}}^{+}=w_{\mathrm{s}}^{\mathrm{f}}\left(x_{\mathrm{s}}^{-}\right),
\end{aligned}\right.
\end{aligned}
$$

where $w_{\mathrm{s}}^{\mathrm{f}}$ is at least continuously differentiable.

\section{Existence And Stability of Periodic ORbits}

The Poincaré return map is a well known tool for determining the existence of periodic orbits and their stability properties; for its use in hybrid systems, see [2], [25]-[27]. This section first defines the Poincaré section and the Poincaré return map that will be used for analyzing periodic orbits 
of (40). Analytical results are then developed that allow a practical means for characterizing stability of certain running gaits.

\section{A. Definition of the Poincaré return map}

Following [2], define the stance-time-to-impact function ${ }^{2}$, $T_{I, \mathrm{~s}}: T \mathcal{Q}_{\mathrm{s}} \rightarrow \mathbb{R} \cup\{\infty\}$, by

$$
T_{I, \mathrm{~s}}:= \begin{cases}\inf \left\{t \geq 0 \mid \varphi_{\mathrm{cl}, \mathrm{s}}\left(t, x_{0}\right) \in S_{\mathrm{s}}^{\mathrm{f}}\right\} & \text { if } \exists t \text { such that } \\ \infty & \varphi_{\mathrm{cl}, \mathrm{s}}\left(t, x_{0}\right) \in S_{\mathrm{s}}^{\mathrm{f}} \\ & \text { otherwise, }\end{cases}
$$

where $\varphi_{\mathrm{cl}, \mathrm{s}}\left(t, x_{0}\right)$ is an integral curve of (24) corresponding to $\varphi_{\mathrm{cl}, \mathrm{s}}\left(0, x_{0}\right)=x_{0}$. From [2, Lemma 3], $T_{I, \mathrm{~s}}$ is continuous at points $x_{0}$ where $0<T_{I, \mathrm{~s}}\left(x_{0}\right)<\infty$ and the intersection with $S_{\mathrm{s}}^{\mathrm{f}}$ is transversal. Hence, $\tilde{\mathcal{X}}_{\mathrm{s}}:=\left\{x_{\mathrm{s}} \in \mathcal{X}_{\mathrm{s}} \mid 0<\right.$ $T_{I, \mathrm{~s}}\left(x_{\mathrm{s}}\right)<\infty$ and $\left.L_{f_{\mathrm{cl}, \mathrm{s}}} H_{\mathrm{s}}^{\mathrm{f}}\left(\varphi_{\mathrm{cl}, \mathrm{s}}\left(T_{I, \mathrm{~s}}\left(x_{\mathrm{s}}\right), x_{\mathrm{s}}\right)\right) \neq 0\right\}$ is open, and consequently, $\tilde{S}_{\mathrm{f}}^{\mathrm{s}}:=\bar{\Delta}_{\mathrm{f}}^{\mathrm{s}}-1\left(\tilde{\mathcal{X}}_{\mathrm{s}}\right)$ is an open subset of $\bar{S}_{\mathrm{f}}^{\mathrm{s}}$. It follows that the generalized Poincare stance map $P_{\mathrm{s}}: \tilde{S}_{\mathrm{f}}^{\mathrm{s}} \rightarrow S_{\mathrm{s}}^{\mathrm{f}}$ defined by

$$
P_{\mathrm{s}}\left(x_{\mathrm{f}}\right):=\varphi_{\mathrm{cl}, \mathrm{s}}\left(T_{I, \mathrm{~s}}\left(\Delta_{\mathrm{f}}^{\mathrm{s}}\left(x_{\mathrm{f}}\right)\right), \Delta_{\mathrm{f}}^{\mathrm{s}}\left(x_{\mathrm{f}}\right)\right),
$$

is well-defined and continuous (the terminology of a generalized-Poincaré map follows Appendix D of [28]).

In analogous fashion, the generalized Poincaré flight map $P_{\mathrm{f}}: \tilde{S}_{\mathrm{s}}^{\mathrm{f}} \rightarrow \tilde{S}_{\mathrm{f}}^{\mathrm{s}}$, is defined by

$$
P_{\mathrm{f}}\left(x_{\mathrm{s}}\right):=\varphi_{\mathrm{cl}, \mathrm{f}}\left(T_{I, \mathrm{f}}\left(\Delta_{\mathrm{s}}^{\mathrm{f}}\left(x_{\mathrm{s}}\right), w_{\mathrm{s}}^{\mathrm{f}}\left(x_{\mathrm{s}}\right)\right), \Delta_{\mathrm{s}}^{\mathrm{f}}\left(x_{\mathrm{s}}\right), w_{\mathrm{s}}^{\mathrm{f}}\left(x_{\mathrm{s}}\right)\right) .
$$

In [28, Appendix D], it is proved that $P_{\mathrm{f}}$ is continuously differentiable. The Poincaré return map $P: \tilde{S}_{\mathrm{s}}^{\mathrm{f}} \rightarrow S_{\mathrm{s}}^{\mathrm{f}}$ for (40) is defined by

$$
P:=P_{\mathrm{s}} \circ P_{\mathrm{f}} .
$$

\section{B. Analysis of the Poincaré return map}

Theorem 1 (Connecting running to walking): Let $P$ be the Poincaré return map defined in (44) for the hyrbid running model in (40). $P$ is also the Poincaré return map for the system with impulse effects

$$
\Sigma_{\mathrm{cl}}:\left\{\begin{aligned}
\dot{x}(t) & =f_{\mathrm{cl}, \mathrm{s}}(x(t)) & & x^{-}(t) \notin S \\
x^{+}(t) & =\Delta\left(x^{-}(t)\right) & & x^{-}(t) \in S,
\end{aligned}\right.
$$

where $S:=\tilde{S}_{\mathrm{s}}^{\mathrm{f}}$ and $\Delta:=\bar{\Delta}_{\mathrm{f}}^{\mathrm{s}} \circ P_{\mathrm{f}}$.

Proof: This follows immediately from the construction of the Poincaré return map in [2, Eq. (14)].

This observation is important because models of the form (45) have been studied in the context of walking gaits. Under certain conditions on the impact map, powerful analysis and feedback design tools have been developed for this class of models [3], [4], and the viability of the feedback designs has been confirmed experimentally [8], [9]. The identification of running with walking indicates how certain results developed for walking may be extended to running. In this section and the next, several results along this line of reasoning are developed and illustrated on an asymptotically stable running gait.

\footnotetext{
${ }^{2}$ Flows from one surface to another are sometimes called impact maps or functions. $T_{I, \mathrm{~s}}$ could also be called the time-to-flight function.
}

Suppose that $\Delta: S \cap Z_{\mathrm{s}} \rightarrow Z_{\mathrm{s}}$, where $Z_{\mathrm{s}}$ is the stancephase zero dynamics manifold. Then, from [3], (45) has a hybrid zero dynamics, which may be called the hybrid zero dynamics of running:

$$
\begin{aligned}
& \dot{z}=f_{\text {zero }}(z) \quad z^{-} \notin S \cap Z_{\mathrm{s}} \\
& z^{+}=\Delta_{\text {zero }}\left(z^{-}\right) \quad z^{-} \in S \cap Z_{\mathrm{s}} \text {, }
\end{aligned}
$$

where the restricted impact map is $\Delta_{\text {zero }}:=\left.\Delta\right|_{S \cap Z_{\mathrm{s}}}$ and $f_{\text {zero }}$ is given by (26). The key properties in walking gaits that led to a rich analytic theory were $Z_{\mathrm{s}}$-invariance, $\Delta: S \cap Z_{\mathrm{s}} \rightarrow Z_{\mathrm{s}}$, and what one may call configuration determinism: $\pi \circ \Delta(S \cap$ $Z_{\mathrm{s}}$ ) consists of a single point, where $\pi: T \mathcal{Q}_{\mathrm{s}} \rightarrow \mathcal{Q}_{\mathrm{s}}$ is the canonical projection. How to achieve these conditions for $\Delta=$ $\bar{\Delta}_{\mathrm{f}}^{\mathrm{s}} \circ P_{\mathrm{f}}$ through design of the flight-phase controller will be detailed in Section VII.

Let $q_{0}^{-}$be as defined in (28) and suppose that $\pi \circ \Delta(S \cap$ $\left.Z_{\mathrm{s}}\right)=\left\{q_{0}^{+}\right\}$. Then (5) can be used to define the positions of the center of mass at the beginning of the stance phase, $\left(x_{\mathrm{cm}}^{+}, y_{\mathrm{cm}}^{+}\right)$, and the end of the stance phase, $\left(x_{\mathrm{cm}}^{-}, y_{\mathrm{cm}}^{-}\right)$. In the following, it is assumed that the center of mass is behind the stance leg a the beginning of the stance phase, and thus, $x_{\mathrm{cm}}^{+}<0$.

Theorem 2 (Characterization of restricted impact map): Suppose that $\Delta: S \cap Z_{\mathrm{s}} \rightarrow Z_{\mathrm{s}}$ and $\pi \circ \Delta\left(S \cap Z_{\mathrm{s}}\right)=\left\{q_{0}^{+}\right\}$. In the coordinates $\left(\theta_{\mathrm{s}}^{-}, \sigma_{1}^{-}\right)$for $S \cap Z_{\mathrm{s}}$, the restricted impact map is given by

$$
\Delta_{\text {zero }}\left(\theta_{\mathrm{s}}^{-}, \sigma_{1}^{-}\right)=\left[\begin{array}{c}
\theta_{\mathrm{s}}^{+} \\
\delta\left(\sigma_{1}^{-}\right)
\end{array}\right],
$$

where

$$
\begin{aligned}
\theta_{\mathrm{s}}^{+} & =\theta_{\mathrm{s}}\left(q_{0}^{+}\right) \\
\delta\left(\sigma_{1}^{-}\right) & =\chi \sigma_{1}^{-}-\sqrt{\left(\beta \sigma_{1}^{-}\right)^{2}+\alpha},
\end{aligned}
$$

and

$$
\begin{aligned}
& \alpha=-2 m^{2} g\left(x_{\mathrm{cm}}^{+}\right)^{2}\left(y_{\mathrm{cm}}^{+}-y_{\mathrm{cm}}^{-}\right) \\
& \beta=m x_{\mathrm{cm}}^{+} \lambda_{y}\left(q_{0}^{-}\right) \\
& \chi=1+m x_{\mathrm{cm}}^{-} \lambda_{y}\left(q_{0}^{-}\right)+m\left(y_{\mathrm{cm}}^{+}-y_{\mathrm{cm}}^{-}\right) \lambda_{x}\left(q_{0}^{-}\right) .
\end{aligned}
$$

The proof is given in the Appendix.

\section{Remark 1:}

1) When $\alpha=0$, that is, the center of mass has the same height at the beginning and end of the stance phase, $\delta\left(\sigma_{1}^{-}\right)=(\chi-|\beta|) \sigma_{1}^{-}$is linear, exactly as in walking.

2) In terms of the coordinates $\left(\theta_{\mathrm{s}}^{-}, \zeta^{-}:=\frac{1}{2}\left(\sigma_{1}^{-}\right)^{2}\right)$, where the (generalized) kinetic energy of the stance-phase zero dynamics is used instead of the angular momentum, the second entry in (47) becomes

$$
\begin{aligned}
& \delta_{e}\left(\zeta^{-}\right)=\left(\chi^{2}+\beta^{2}\right) \zeta^{-} \\
& \quad-\chi \sqrt{2 \alpha \zeta^{-}+\left(2 \beta \zeta^{-}\right)^{2}}+\frac{\alpha}{2} .
\end{aligned}
$$

3) Implicit in the construction of $S:=\tilde{S}_{\mathrm{s}}^{\mathrm{f}}$ is the condition $2 \alpha \zeta^{-}+\left(2 \beta \zeta^{-}\right)^{2} \geq 0$. Also a part of the construction of $S$ is the condition that $T_{I, \mathrm{f}}$ is a positive real number; under the assumptions made on $\Delta$, this is equivalent to checking that $y_{\mathrm{cm}}^{+}>y_{\mathrm{cm}}^{-}$and $\lambda_{y}\left(q_{0}^{-}\right)<0$ do not simultaneously occur. 
Let $P: S \rightarrow S$ be the Poincaré return map for (45), and hence, also for (40), and suppose that $\Delta: S \cap Z_{\mathrm{s}} \rightarrow Z_{\mathrm{s}}$. Then $P: S \cap Z_{\mathrm{s}} \rightarrow S \cap Z_{\mathrm{s}}$. Define the restricted Poincaré return $\operatorname{map} \rho: S \cap Z_{\mathrm{s}} \rightarrow S \cap Z_{\mathrm{s}}$ by

$$
\rho:=\left.P\right|_{S \cap Z_{\mathrm{s}}}
$$

The restricted Poincare return map is important because it is scalar and, by [2, Theorem 2] (see [3, Sec. IV]), asymptotically stable fixed points of it correspond to asymptotically stable periodic orbits of the hybrid model (45), and hence, to asymptotically stable running gaits.

Theorem 3 (Closed-form for $\rho$ ): Suppose that $\Delta: S \cap$ $Z_{\mathrm{s}} \rightarrow Z_{\mathrm{s}}$ and $\pi \circ \Delta\left(S \cap Z_{\mathrm{s}}\right)=\left\{q_{0}^{+}\right\}$. Let $\left(\theta_{\mathrm{s}}^{-}, \sigma_{1}^{-}\right) \in S \cap Z_{\mathrm{s}}$, and set $\zeta^{-}:=\frac{1}{2}\left(\sigma_{1}^{-}\right)^{2}$. Then

$$
\begin{aligned}
\rho\left(\zeta^{-}\right)= & \left(\chi^{2}+\beta^{2}\right) \zeta^{-} \\
& -\chi \sqrt{2 \alpha \zeta^{-}+\left(2 \beta \zeta^{-}\right)^{2}}+\frac{\alpha}{2}-V_{\text {zero }}\left(\theta_{\mathrm{s}}^{-}\right),
\end{aligned}
$$

with domain of definition

$$
\begin{aligned}
\mathcal{D}_{\rho}:=\left\{\zeta^{-}>0 \mid \delta_{e}\left(\zeta^{-}\right)-\right. & V_{\mathrm{zero}}^{\max }>0, \\
& \left.2 \alpha \zeta^{-}+\left(2 \beta \zeta^{-}\right)^{2} \geq 0\right\},
\end{aligned}
$$

where $\delta_{e}$ is defined in (50), and

$$
V_{\mathrm{zero}}^{\max }:=\max _{\theta_{\mathrm{s}}^{+} \leq \theta_{\mathrm{s}} \leq \theta_{\mathrm{s}}^{-}} V_{\text {zero }}\left(\theta_{\mathrm{s}}\right)
$$

Moreover, the first derivative of the restricted Poincaré return map is

$$
\frac{d \rho}{d \zeta^{-}}\left(\zeta^{-}\right)=\left(\chi^{2}+\beta^{2}\right)-\chi \frac{\alpha+4 \beta^{2} \zeta^{-}}{\sqrt{2 \alpha \zeta+\left(2 \beta \zeta^{-}\right)^{2}}} .
$$

The proof is given in the Appendix. The following corollary is immediate.

\section{Corollary 1 (Exponentially stable fixed points):}

Suppose that $\zeta^{*} \in \mathcal{D}_{\rho}$ is a fixed point of $\rho$. Then it is exponentially stable if, and only if,

$$
\mu:=\left(\chi^{2}+\beta^{2}\right)-\chi \frac{\alpha+4 \beta^{2} \zeta^{*}}{\sqrt{2 \alpha \zeta^{*}+\left(2 \beta \zeta^{*}\right)^{2}}}
$$

satisfies $|\mu|<1$.

\section{ILLUSTRATION ON RABBIT}

Using the method proposed in [11], a time-trajectory of (18), corresponding to an average running speed of $1.5 \mathrm{~m} / \mathrm{s}$, was determined for RABBIT (see [1] for details on the planar, bipedal robot, RABBIT). A stick-figure diagram corresponding to the running motion is given in [12]. Denote by $\mathcal{O}$ the path traced out by this trajectory in the state spaces of the hybrid model of the robot. $\mathcal{O}$ intersects $S_{\mathrm{s}}^{\mathrm{f}}$ and $S_{\mathrm{f}}^{\mathrm{s}}$ exactly once each; define $x_{\mathrm{f}}^{*}=\mathcal{O} \cap S_{\mathrm{f}}^{\mathrm{s}}$ and $x_{\mathrm{s}}^{*}=\mathcal{O} \cap S_{\mathrm{s}}^{\mathrm{f}}$. The goal is to design a timeinvariant state-feedback controller à la Section $\mathrm{V}$ that has $\mathcal{O}$ as its asymptotically-stable periodic orbit. Recall that designing the controller is equivalent to specifying the output functions in (21) and (34) and the parameter update-law in (40).

\section{A. Stance Phase Controller Design}

On the stance phase of the running trajectory, $\theta_{\mathrm{s}}$ varies between $\theta_{\mathrm{s}}^{+}=1.2758 \mathrm{rad}$ and $\theta_{\mathrm{s}}^{-}=1.8849 \mathrm{rad}$. As in [5], an output $y_{\mathrm{s}}=h_{\mathrm{s}}(q):=q_{\mathrm{b}}-h_{d, \mathrm{~s}} \circ \theta_{\mathrm{s}}(q)$ is designed so that it vanishes (nearly) along the stance phase of the periodic orbit, and thus the orbit will be an integral curve of the stance-phase zero dynamics. For this, the function $h_{d, \mathrm{~s}}$ was selected to be a fourth-order polynomial in $\theta_{\mathrm{s}}$. The design method in [11] that is used to compute the periodic orbit essentially guarantees that the technical conditions of Section $\mathrm{V}$ are satisfied for $h_{\mathrm{s}}$; nevertheless, the conditions are formally verified. Once $h_{\mathrm{s}}$ is known, so is $Z_{\mathrm{s}}$, and, by construction, $\mathcal{O} \cap T \mathcal{Q}_{\mathrm{s}} \subset Z_{\mathrm{s}}$. Define $S_{\mathrm{s}}^{\text {init }}=\left\{(q, \dot{q}) \mid \theta_{\mathrm{s}}(q)=\theta_{\mathrm{s}}^{+}\right\}$.

\section{B. Stability of the periodic orbits}

The data required to determine the restricted Poincaré map $\rho$ in Theorem 3 can be computed directly from: $h_{d, \mathrm{~s}}$. This was done and yielded $\alpha=27.3270, \beta=-0.0129, \chi=0.9549$, and $V_{\text {zero }}=-257.68$. Computing $\rho$ results in $\zeta^{*}=801.5$ and $\mu=0.7855$. Since $\mu<1$, if a flight-phase controller can be determined to meet the conditions of Theorem 3, the orbit will be asymptotically stable. A plot of the restricted Poincaré map is provided in [12].

\section{Flight Phase Controller Design}

The flight phase controller, $y_{\mathrm{f}}=h_{\mathrm{f}}\left(q_{\mathrm{f}}, a_{\mathrm{f}}\right):=q_{\mathrm{b}}-$ $h_{d, \mathrm{f}}\left(x_{\mathrm{cm}}, a_{\mathrm{f}}\right), a_{\mathrm{f}}=w_{\mathrm{s}}^{\mathrm{f}}\left(x_{\mathrm{s}}^{-}\right)$, is to be designed so that $\Delta(S \cap$ $\left.Z_{\mathrm{s}}\right) \subset Z_{\mathrm{s}}$ and $\pi \circ \Delta\left(S \cap Z_{\mathrm{s}}\right)$ is a single point. These two conditions will hold if, and only if,

$$
\Delta\left(S \cap Z_{\mathrm{s}}\right) \subset Z_{\mathrm{s}} \cap S_{\mathrm{s}}^{\text {init }} .
$$

Analogously to (28) and (29), $Z_{\mathrm{s}} \cap S_{\mathrm{s}}^{\text {init }}$ is given by

$$
\left\{\left(q_{0}^{+}, \dot{q}^{+}\right) \mid q_{0}^{+}=\Phi_{\mathrm{s}}^{-1}\left(0, \theta_{\mathrm{s}}^{+}\right), \quad \dot{q}^{+}=\dot{q}_{0}^{+} \sigma_{1}^{+}, \quad \sigma_{1}^{+} \in \mathbb{R}\right\},
$$

where

$$
\dot{q}_{0}^{+}=\left.\left[\begin{array}{c}
\frac{\partial h_{\mathrm{s}}}{\partial q} \\
A_{5}
\end{array}\right]^{-1}\right|_{q=q_{0}^{+}}\left[\begin{array}{l}
0 \\
1
\end{array}\right] .
$$

From Theorem 2, it follows that (56) is equivalent to

$$
\Delta\left(q_{0}^{-}, \dot{q}_{0}^{-} \sigma_{1}^{-}\right)=\left(q_{0}^{+}, \dot{q}_{0}^{+} \delta\left(\sigma_{1}^{-}\right)\right),
$$

which gives specific boundary conditions, just after impact, to be met by the design of the flight phase controller. In particular, recalling that $q=\left(q_{b}^{\prime}, q_{5}\right)^{\prime}$, it is seen that (59) places constraints on the body configuration variables, their derivatives, and $q_{5}$, while the constraint on $\dot{q}_{5}$ is equivalent to $\sigma_{1}^{+}=\delta\left(\sigma_{1}^{-}\right)$, if the other constraints are met.

For the purpose of computation, it is convenient to transform (59) to conditions in $T \mathcal{Q}_{\mathrm{f}}$ instead of $T \mathcal{Q}_{\mathrm{s}}$. This is done as follows: the boundary conditions (59) specify the height of the center of mass at impact, and from this information, the flight time, $t_{\mathrm{f}}$, is computed for any initial condition in $S \cap Z_{\mathrm{s}}$; see (67) in the Appendix. Using (68) and the impact model, a function $\dot{\bar{q}}_{0}\left(q_{0}^{+}, \sigma_{1}^{-}\right)$is determined such that (59) is equivalent to

$$
\left(q_{0}^{-}, \dot{q}_{0}^{-} \sigma_{1}^{-}\right) \mapsto\left(R^{-1} q_{0}^{+}, \dot{\bar{q}}_{0}\left(q_{0}^{+}, \sigma_{1}^{-}\right)\right) .
$$


The design of $h_{d, f}$ can now be given in two steps. First, define

$$
\tau\left(x_{\mathrm{cm}}, \sigma_{1}^{-}\right)=\frac{x_{\mathrm{cm}}-x_{\mathrm{cm}}^{-}}{t_{\mathrm{f}} \dot{x}_{\mathrm{cm}}^{-}}=\frac{x_{\mathrm{cm}}-x_{\mathrm{cm}}^{-}}{t_{\mathrm{f}} \lambda_{x}\left(q_{0}^{-}\right) \sigma_{1}^{-}} ;
$$

the real-valued function $\tau$ varies between 0 and 1 and can be used to parameterize trajectories from $S \cap Z_{\mathrm{s}}$ to $S_{\mathrm{s}}^{\text {init }} \cap Z_{\mathrm{s}}$ in a neighborhood of the periodic orbit. Choose a function $\operatorname{poly}\left(\mathrm{a}_{0}, \cdots, \mathrm{a}_{4}\right):[0,1] \rightarrow \mathrm{R}^{4}$ such that

$$
\begin{aligned}
\operatorname{poly}\left(\mathrm{a}_{0}, \cdots, \mathrm{a}_{4}\right)(0) & =a_{1} \\
\frac{d \text { poly }}{d \tau}\left(a_{0}, \cdots, a_{4}\right)(0) & =a_{2} \\
\operatorname{poly}\left(\mathrm{a}_{1}, \cdots, \mathrm{a}_{4}\right)(1) & =a_{3} \\
\frac{d \text { poly }}{d \tau}\left(a_{0}, \cdots, a_{4}\right)(1) & =a_{4},
\end{aligned}
$$

and there exist $a_{1}^{*}, \cdots, a_{4}^{*}$ for which $q_{b}-\operatorname{poly}\left(\mathrm{a}_{0}^{*}, \cdots, \mathrm{a}_{4}^{*}\right)(\tau)$ (nearly) vanishes on $\mathcal{O}$. Here, this was accomplished with a fourth order polynomial. Off of the orbit, use (62) to solve for $a_{1}, \cdots, a_{4}$ as functions of $\sigma_{1}^{-}$so that $q_{b}(\tau)=$ poly $\left(\mathrm{a}_{0}, \cdots, \mathrm{a}_{4}\right)(\tau)$ satisfies the constraints on the body coordinates imposed by (60). Specifically, set $a_{1}=\left(q_{0}^{-}\right)_{b}$, $a_{3}=\left(R^{-1} q_{0}^{+}\right)_{b}, a_{2}=\left(\dot{q}_{0}^{-} \sigma_{1}^{-}\right)_{b}$, and $a_{4}=\left(\dot{\bar{q}}_{0}\left(q_{0}^{+}, \sigma_{1}^{-}\right)\right)_{b}$. Define

$$
h_{d, \mathrm{f}}\left(x_{\mathrm{cm}}, \sigma_{1}^{-}, a_{0}\right):=\operatorname{poly}\left(\mathrm{a}_{0}, \ldots, \mathrm{a}_{4}\right)(\tau)
$$

with $a_{i}\left(\sigma_{1}^{-}\right), i=1, \ldots, 4$ and $\tau\left(x_{\mathrm{cm}}, \sigma_{1}^{-}\right)$. Define $q_{5}(0)=$ $\left(q_{0}^{-}\right)_{5}$ and $q_{5}^{d}=\left(R^{-1} q_{0}^{+}\right)_{5}$.

In the second step, the goal is to select $a_{0}$ as a function of $\sigma_{1}^{-}$so that the $q_{5}$-component satisfies the constraints. This is done as follows. The output (63) satisfies all of the conditions of Section V, and hence the evolution of $q_{5}$ in the flight-phase zero dynamics is given by $\dot{q}_{5}=\kappa_{1, \mathrm{f}}\left(\sigma_{\mathrm{cm}}, x_{\mathrm{cm}}, \dot{x}_{\mathrm{cm}}, \sigma_{1}^{-}, a_{0}\right)$. In the flight phase, $\sigma_{\mathrm{cm}}$ and $\dot{x}_{\mathrm{cm}}$ are constant and can be substituted by their values from $\left(S \cap Z_{\mathrm{s}}\right)$. In addition, $x_{\mathrm{cm}}(t)=x_{\mathrm{cm}}^{-}+t \lambda_{x}\left(q_{0}^{-}\right) \sigma_{1}^{-}$. Hence, $\dot{q}_{5}=\tilde{\kappa}_{1, \mathrm{f}}\left(t, \sigma_{1}^{-}, a_{0}\right)$. Letting $\sigma_{1}^{-*}$ denote the value of $\sigma_{1}^{-}$on the $\mathcal{O}, q_{5}^{d}=q_{5}(0)+$ $\int_{0}^{t_{f}} \tilde{\kappa}_{1, \mathrm{f}}\left(t, \sigma_{1}^{-*}, a_{0}^{*}\right) d t$ is satisfied because, by construction of the output, the orbit corresponds to an integral curve of the flight-phase zero dynamics. Finally, it is verified (numerically) that

$$
\left.\frac{\partial}{\partial a_{0}}\left(q_{5}^{d}-q_{5}(0)-\int_{0}^{t_{f}} \tilde{\kappa}_{1, \mathrm{f}}\left(t, \sigma_{1}^{-*}, a_{0}\right) d t\right)\right|_{a_{0}=a_{0} *} \neq 0
$$

and thus by the implicit function theorem, there exists an open subset about $\sigma_{1}^{-*}$ and a differentiable function $\tilde{w}_{\mathrm{s}}^{\mathrm{f}}$ such that $\tilde{w}_{\mathrm{s}}^{\mathrm{f}}\left(\sigma_{1}^{-*}\right)=a_{0}^{*}$ and

$$
q_{5}^{d}=q_{5}(0)+\int_{0}^{t_{f}} \tilde{\kappa}_{1, \mathrm{f}}\left(t, \sigma_{1}^{-}, \tilde{w}_{\mathrm{s}}^{\mathrm{f}}\left(\sigma_{1}^{-}\right)\right) d t .
$$

Since (65) is scalar while $a_{0}$ has four components, there exist an infinite number of solutions for $\tilde{w}_{\mathrm{s}}^{\mathrm{f}}$. Hence, a numerical optimization was performed to find, for each point in a neighborhood of $\sigma_{1}^{*}$, a value of $a_{0}$ that steers $q_{5}$ to $q_{5}^{d}$, while minimizing $\left\|a_{0}-a_{0}^{*}\right\|$. The flight-phase control design is completed by formally defining $h_{d, \mathrm{f}}\left(q_{\mathrm{f}}, a_{\mathrm{f}}\right), a_{\mathrm{f}}:=\left(\sigma_{1}^{-}, a_{0}^{\prime}\right)^{\prime}$, and $w_{\mathrm{s}}^{\mathrm{f}}\left(x_{\mathrm{s}}^{-}\right):=\left(\sigma_{1}^{-}, \tilde{w}_{\mathrm{s}}^{\mathrm{f}}\left(\sigma_{1}^{-}\right)^{\prime}\right)^{\prime}$.

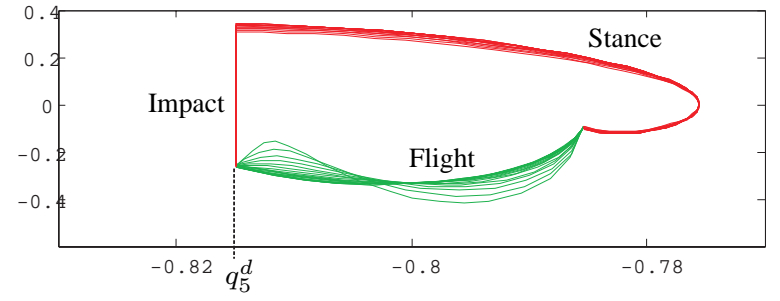

Fig. 2. The torso angle ( $\mathrm{x}$-axis) in units of radians versus its velocity (y-axis) in units of radians per second in the stance and flight phases. Notice that the flight-phase controller has regulated the torso angle to its desired value of $q_{5}^{d}$ at impact. The plot indicates that a limit cycle is achieved. In fact, the obtained limit cycle corresponds to the original periodic orbit, $\mathcal{O}$.

\section{Simulation}

The controller has been simulated on a model of RABBIT. Assuming no modeling error and initializing the closedloop system off of the periodic orbit-with an error in the velocity-yields the simulation data presented in Fig. 2. Additional plots and animations of the running motion are available at [12]. The robustness of the controller is being evaluated on the compliant contact model used in [5]. The preliminary results are encouraging.

\section{CONCLUSIONS}

A time-invariant feedback control strategy has been developed for a bipedal runner. The flight-phase portion of the control strategy was designed so as to create a generalized impact map with properties similar to those of the impact maps that occur in models of walking. This led to the deliberate design of a hybrid zero dynamics of running, that is, a lowdimensional, invariant, sub-dynamic of the closed-loop hybrid system. Asymptotically stable orbits of the hybrid zero dynamics are asymptotically stabilizable orbits of the full-order hybrid model. Using the idea of a restricted Poincaré return map-that is, the Poincaré return map restricted to the hybrid zero dynamics - an explicit criterion for the existence of a periodic orbit was given, as well as an explicit characterization of its stability properties.

The principal results were illustrated on a five-link, fouractuator planar biped with revolute joints. A periodic trajectory computed in [11] was interpreted as a desired periodic orbit in the state space of the robot model. A time-invariant feedback controller was designed to realize this orbit as an attractive solution of the hybrid zero dynamics, without, it is very important to note, the use of trajectory tracking. Very roughly speaking, the controller is "clocked" to events on the orbit and not to time. Hence, when perturbed away from the orbit, the robot's links regain "synchrony" with respect to the robot's position on the orbit and not with respect to time.

\section{ACKNOWLEDGMENTS}

The work of J.W. Grizzle was supported by NSF grant ECS0322395 . 


\section{APPENDIX}

\section{A. Proof of Theorem 2}

By (28), points in $S \cap Z_{\mathrm{s}}$ are parameterized by $\left(q_{0}^{-}, \dot{q}_{0}^{-} \sigma_{1}^{-}\right)$. The position of the center of mass $\left(x_{\mathrm{cm}}^{-}, y_{\mathrm{cm}}^{-}\right)$is obtained by evaluating (5) at $q_{0}^{-}$and its velocity is obtained from (33), $\left(\dot{x}_{\mathrm{cm}}^{-}, \dot{y}_{\mathrm{cm}}^{-}\right)=\left(\lambda_{x}\left(q_{0}^{-}\right) \sigma_{1}^{-}, \lambda_{y}\left(q_{0}^{-}\right) \sigma_{1}^{-}\right)$. The angular momentum about the center of mass can be determined from (16) to be

$$
\sigma_{\mathrm{cm}}^{-}=\sigma_{1}^{-}-m\left(y_{\mathrm{cm}}^{-} \lambda_{x}\left(q_{0}^{-}\right) \sigma_{1}^{-}-x_{\mathrm{cm}}^{-} \lambda_{y}\left(q_{0}^{-}\right) \sigma_{1}^{-}\right) .
$$

Since the transition map from the stance phase to the flight phase preserves positions and velocities, (66) is also the angular momentum at the beginning of the flight phase, and because angular momentum is conserved during ballistic motion, (66) is also the value of the angular momentum at the end of the flight phase. From the hypotheses $\Delta: S \cap Z_{\mathrm{s}} \rightarrow Z_{\mathrm{s}}$ and $\pi \circ \Delta\left(S \cap Z_{\mathrm{s}}\right)$ is a single point, the position of the center of mass at the end of the flight phase is known and equal to the position of the center of mass at the beginning of the subsequent stance phase, which is given by $\left(x_{\mathrm{cm}}^{+}, y_{\mathrm{cm}}^{+}\right)$. From this, the flight time, $t_{f}$, can be computed

$$
t_{f}=\frac{\dot{y}_{\mathrm{cm}}^{-}}{g}+\frac{\sqrt{\left(\dot{y}_{\mathrm{cm}}^{-}\right)^{2}-2 g\left(y_{\mathrm{cm}}^{+}-y_{\mathrm{cm}}^{-}\right)}}{g},
$$

and from (15), the velocity of the center of mass at the end of the flight phase is determined

$$
\left[\begin{array}{c}
\dot{x}_{\mathrm{cm}}\left(t_{f}\right) \\
\dot{y}_{\mathrm{cm}}\left(t_{f}\right)
\end{array}\right]=\left[\begin{array}{c}
\dot{x}_{\mathrm{cm}}^{-} \\
-\sqrt{\left(\dot{y}_{\mathrm{cm}}^{-}\right)^{2}-2 g\left(y_{\mathrm{cm}}^{+}-y_{\mathrm{cm}}^{-}\right)}
\end{array}\right] .
$$

Equations (66), (68), (16) allow the angular momentum about the contact point at the end of the flight phase, $\sigma_{2}^{-}$, to be evaluated, and then (17) allows the evaluation of the angular momentum about the stance leg at the beginning of the subsequence stance phase. This yields

$$
\begin{gathered}
\sigma_{1}^{+}=\sigma_{1}^{-}-m\left(y_{\mathrm{cm}}^{-} \lambda_{x}\left(q_{0}^{-}\right) \sigma_{1}^{-}-x_{\mathrm{cm}}^{-} \lambda_{y}\left(q_{0}^{-}\right) \sigma_{1}^{-}\right)+ \\
m y_{\mathrm{cm}}^{+} \lambda_{x}\left(q_{0}^{-}\right) \sigma_{1}^{-}+m x_{\mathrm{cm}}^{+} \sqrt{\left(\lambda_{y}\left(q_{0}^{-}\right) \sigma_{1}^{-}\right)^{2}-2 g\left(y_{\mathrm{cm}}^{+}-y_{\mathrm{cm}}^{-}\right)},
\end{gathered}
$$

which, after simplification, completes the proof.

\section{B. Proof of Theorem 3}

From [3, Sec. IV], in the coordinates $\left(\theta_{\mathrm{s}}, \zeta=\frac{1}{2}\left(\sigma_{1}\right)^{2}\right)$ for $Z_{\mathrm{s}}$, the stance-phase zero dynamics can be integrated as $\zeta\left(\theta_{\mathrm{s}}\right)=\zeta^{+}-V_{\text {zero }}\left(\theta_{\mathrm{s}}\right)$. Evaluating at $\theta_{\mathrm{s}}^{-}$and applying (50) yields $\rho\left(\zeta^{-}\right)=\delta_{e}\left(\zeta^{-}\right)-V_{\text {zero }}\left(\theta_{\mathrm{s}}^{-}\right)$. The domain of $\rho$ follows from [3, Thm. 3].

\section{REFERENCES}

[1] C. Chevallereau, G. Abba, Y. Aoustin, E. Plestan, F. Westervelt, C. Canduas-de Wit, and J. Grizzle, "RABBIT: A testbed for advanced control theory," IEEE Control Systems Magazine, vol. 23, no. 5, pp. 57-79, October 2003.

[2] J. Grizzle, G. Abba, and F. Plestan, "Asymptotically stable walking for biped robots: Analysis via systems with impulse effects," IEEE Trans. on Automatic Control, vol. 46, pp. 51-64, January 2001.
[3] E. Westervelt, J. Grizzle, and D. Koditschek, "Hybrid zero dynamics of planar biped walkers," IEEE Trans. on Automatic Control, vol. 48, no. 1, pp. 42-56, January 2003.

[4] E. Westervelt, J. Grizzle, and C. Canudas-de-Wit, "Switching and PI control of walking motions of planar biped walkers," IEEE Trans. on Automatic Control, vol. 48, no. 2, pp. 308-312, February 2003.

[5] F. Plestan, J. Grizzle, E. Westervelt, and G. Abba, "Stable walking of a 7-DOF biped robot," IEEE Trans. on Robotics and Automation, vol. 19, no. 4, pp. 653-668, August 2003.

[6] C. Chevallereau, "Time scaling control for an underactuated biped robot," IEEE Trans. on Robotics and Automation, vol. 18, April 2003.

[7] C. Chevallereau, A. Formal'sky, and D. Djoudi, "Tracking of a joint path for the walking of an underactuated biped," Robotica, vol. 22, pp. 15-28, 2004.

[8] E. Westervelt, G. Buche, and J. Grizzle, "Experimental validation of a framework for the design of controllers that induce stable walking in planar bipeds," Int. Journal of Robotics Research, to appear. See [29] for a preprint. Submitted in September 2003.

[9] — - "Inducing dynamically stable walking in an underactuated prototype planar biped," in Proc. of the IEEE Int. Conf. on Robotics and Automation, May 2004, to appear. See [29] for a preprint.

[10] C. Chevallereau and P. Sardain, "Design and actuation optimization of a 4 axes biped robot for walking and running," in Proc. of the IEEE Int. Conf. on Robotics and Automation, April 2000, pp. 3365-3370.

[11] C. Chevallereau and Y. Aoustin, "Optimal reference trajectories for walking and running of a biped robot," Robotica, vol. 19, no. 5, pp. 557-569, September 2001.

[12] Supplemental material for this article, "available at [30]," March 2004.

[13] M. Raibert, Legged robots that balance. Mass.: MIT Press, 1986.

[14] _ , "Legged robots," Communications of the ACM, vol. 29, no. 6, pp. 499-514, 1986.

[15] D. Koditschek and M. Buehler, "Analysis of a simplified hopping robot," Int. Journal of Robotics Research, vol. 10, no. 6, pp. 587-605, 1991.

[16] M. Bühler, D. E. Koditschek, and P. J. Kindlmann, "Planning and control of a juggling robot," Int. Journal of Robotics Research, vol. 13, no. 2, pp. 101-118, 1994.

[17] C. Francois and C. Samson, "A new approach to the control of the planar one-legged hopper," Int. Journal of Robotics Research, vol. 17, no. 11, pp. 1150-1166, 1998 .

[18] M. Ahmadi and M. Buehler, "Stable control of a simulated one-legged running robot with hip and leg compliance," IEEE Trans. on Robotics and Automation, vol. 13, no. 1, pp. 96-104, February 1997.

[19] J. Hodgins and M. Raibert, "Adjusting step length for rough terrain locomotion," IEEE Trans. on Robotics and Automation, vol. 7(3), pp. 289-298, June 1991.

[20] "Johnnie - The TUM Biped Walking Robot," http://www.amm.mw. tu-muenchen.de/misc/messe/hanmesse-e.html, January 2003.

[21] F. Pfeiffer, K. Loffler, and M. Gienger, "The concept of jogging johnnie," in Proc. of the IEEE Int. Conf. on Robotics and Automation, May 2002.

[22] "ROBEA: Robotics and Artificial Entities," http://robot-rabbit.lag. ensieg.inpg.fr/index.php (in French; for an English version, click on the British flag), February 2004.

[23] Y. Hurmuzlu and D. Marghitu, "Rigid body collisions of planar kinematic chains with multiple contact points," Int. Journal of Robotics Research, vol. 13, no. 1, pp. 82-92, 1994.

[24] J. Guckenheimer and S. Johnson, "Planar hybird systems," in Hybrid Systems II, Lecture Notes in Computer Science. Springer-Verlag, 1995, pp. 203-225.

[25] B. Espiau and A. Goswani, "Compass gait revisited," in Proc. of the IFAC Symposium on Robot Control, Capri, Italy, September 1994, pp. 839-846.

[26] I. Hiskens, "Stability of hyrbid limit cycles: application to the compass gait biped robot," in IEEE CDC, December 2001, pp. 774-779.

[27] S. Nersesov, V. Chellaboian, and W. Haddad, "A generalization of Poincaré's theorem to hybrid and impulsive dynamical systems," Int. J. Hybrid Systems, vol. 2, pp. 35-51, 2002.

[28] T. Parker and L. Chua, Practical Numerical Algorithms for Chaotic Systems. New York: Springer-Verlag, 1989.

[29] E. Westervelt, "Eric Westervelt's publications," 2004, http://www. mecheng.osu.edu/ $\sim$ westerve/publications/.

[30] J. Grizzle, "Publications on robotics and control," http://www.eecs. umich.edu/ grizzle/papers/robotics.html, February 2004. 